\title{
Biopsy and rebiopsy for non-small-cell lung cancer: current and future methods
}

\author{
Paul Zarogoulidis*,1, Christoforos Kosmidis ${ }^{1}$, Varvara Fyntanidou ${ }^{2}$, Zoi Aidoni ${ }^{1}$, Kosmas \\ Tsakiridis $^{3}$, Charilaos Koulouris ${ }^{1}$, Nikolaos Michalopoulos ${ }^{1}$, Anastasios Barmpas ${ }^{1}$, Haidong \\ Huang ${ }^{4}$, Chong Bai ${ }^{4}$, Wolfgang Hohenforst-Schmidt ${ }^{5}$ \& Konstantinos Sapalidis ${ }^{1}$ \\ ${ }^{1}$ Third Department of Surgery, 'AHEPA' University Hospital, Aristotle University of Thessaloniki, Medical School, Thessaloniki, Greece \\ ${ }^{2}$ Anesthesiology Department, 'AHEPA' University Hospital, Aristotle University of Thessaloniki, Medical School, Thessaloniki, Greece \\ ${ }^{3}$ Thoracic Surgery Department, 'Interbalkan' European Medical Center, Thessaloniki, Greece \\ ${ }^{4}$ Department of Respiratory \& Critical Care Medicine, Changhai Hospital, the Second Military Medical University, Shanghai, China \\ ${ }^{5}$ Sana Clinic Group Franken, Department of Cardiology/Pulmonology/Intensive Care/Nephrology, 'Hof' Clinics, University of \\ Erlangen, Hof, Germany \\ *Author for correspondence: pzarog@hotmail.com
}

\begin{abstract}
"Rapid on-site evaluation is another technique that provides information within the biopsy procedure, regarding whether the material that we obtained was sufficient for diagnosis."
\end{abstract}

First draft submitted: 25 August 2019; Accepted for publication: 10 September 2019; Published online: 17 October 2019

Keywords: bronchoscopy • cone beam CT • convex probe endobronchial ultrasound • CT-guided biopsy • electromagnetic navigation • esophageal ultrasound • non-small-cell lung cancer $\bullet$ PET-CT • radial endobronchial ultrasound • transthoracic ultrasound

Lung cancer is still predominately a disease with late-stage diagnosis and is the second highest cause of cancer death, after prostate cancer for males and breast cancer for females. In the last 5 years, there has been an effort to focus on prevention for smokers, with several lung cancer screening protocols being evaluated. In the last decade, the pharmaceutical industry has continued to investigate the genome of non-small-cell lung cancer and new emerging targeted treatments have been developed as a result. These novel therapies that have been developed were based on: EGFR mutation, anaplastic lymphoma kinase mutation, proto-oncogene $B-R a f(B R A F)$, proto-oncogene tyrosineprotein kinase ROS-1 (ROS1) and PD-L1. Tyrosine kinase inhibitors (TKIs) were developed as drugs for EGFR, anaplastic lymphoma kinase mutation, ROS1- and BRAF-based therapies and immunotherapy was developed for PD-L1-based therapy [1-5]. There are currently different types of TKIs used as first-line treatment. However, these drugs can cause side effects including colitis, rashes or pneumonitis. These adverse effects have been shown to be associated with the effectiveness of the therapy. The more intense the adverse effects, the greater the resulting tumor reduction and metastasis control. Currently, we can adjust the dosage so that the adverse effects are minimized while also ensuring the therapy remains efficient. Moreover, drug resistance is usually observed in EGFR-positive patients when using TKIs because the mutation T790M is developed [1]. However, novel TKIs have been developed in order to overcome this. The main issue, until now, has been determining how to obtain the best possible sample that harbors the mutation. This can be achieved with tissue re-biopsy, or by blood sample [2,3]. Each method has its advantages and disadvantages. Re-biopsy is performed with an invasive technique, while a blood sample is easier to obtain. However, only with a tissue sample we can be $100 \%$ sure that a T790M can be found [3]. Immunotherapy can be administered alone as first-line treatment if PD-L1 expression $\geq 50 \%$ but it can also be administered as second-line treatment, indifferent of the PD-L1 expression [6]. Today, we can also administer immunotherapy as first-line treatment in combination with chemotherapy, indifferent of the PD-L1 expression [7]. Immunotherapy also has adverse effects, such as pneumonitis, orogonitis, vitiligo, dysregulation of the thyroid gland and hepatitis resurrection $[8,9]$. There have been cases where the tumor in patients with very high PD-L1 expression $(\geq 90 \%)$ induced tumor necrosis to such a degree that the tumor melted within $24 \mathrm{~h} \mathrm{[10].} \mathrm{Before} \mathrm{assessing} \mathrm{the} \mathrm{genome} \mathrm{in} \mathrm{a}$ tissue sample, cells or blood, we should comment on the methods of biopsy and how to properly select for each patient. 
Pulmonary physicians had the bronchoscope for many years before the evolution of the ultrasound systems. For peripheral nodules, we can use the radial endobronchial ultrasound (EBUS), potentially combined with a C-arm in order to enhance the efficiency of the method [11,12]. Before the radial-EBUS, we used a C-arm for guidance of the biopsy forceps within the pulmonary parenchyma. However, this method was only efficient for large masses $(\geq 3 \mathrm{~cm})$. For pulmonary nodules, we can use electromagnetic navigation. There are currently different guidance systems on the market and, again, the addition of radial-EBUS and/or C-arm can enhance the efficiency of the electromagnetic navigation technique [11]. Cone beam computed tomography (CT) is another real-time navigation system that can be used with very high efficiency, by both the pulmonary physicians or the radiologists for masses and nodules [13,14]. Transthoracic ultrasound is another type of equipment that can be used either by the pulmonary physicians or the radiologists for peripheral large masses. CT-guided biopsy is another method for biopsy, but has limitations when compared with cone beam CT. Cone beam CT is real-time navigation, CT-guided biopsy is not. Moreover, neither of these techniques should be used for central lesions next to large vessels, for safety reasons. The real evolution for lung cancer staging became a reality with the convex probe EBUS (CP-EBUS) [15]. This endoscope can access those lymph node stations, which are proposed from the International Association for the Study of Lung Cancer (IALSC; CO, USA), within the mesothorax for proper lung cancer staging. It can safely puncture lymph node stations next to large vessels and, of course, access central masses which are surrounding central airways. The esophageal ultrasound (EUS) can be used to access lymph node stations like 7 and $4 \mathrm{~L}$ in the mediastinum, and every mass that is located paraoesophageal. In the case where a lymph node station within the mediastinum is so enlarged that it is touching the esophagus, it can be punctured with the EUS. Similarly, for CP-EBUS there are cases where a mass can be accessed with using the CP-EBUS through the esophagus. In some countries, the CP-EBUS is used to take biopsy from the left adrenal gland, especially in short patients $(\leq 1.70 \mathrm{~cm})$. The bronchoscope and CP-EBUS can use 19, 21 and 22G needles in order to acquire a sample. The larger the needle diameter, the larger the tissue sample. However, we should not forget that we cannot use the 19G needle in all lesions, since this could have serious adverse effects. Since the 19G needle has a larger diameter, it cannot be used to take biopsy from lymph node stations which are in close contact with large vessels. However, a 19G needle can be used for the EUS.

For CT-guided biopsy, transthoracic ultrasound-guided biopsy and cone beam CT-guided biopsy, we can use larger core biopsy needles like 16 and 18G. Pneumothorax and hemothorax can occur in any case where needles are used. Forceps and specially designed brushes can also be used through endoscopes for endobronchial lesions. A very important factor is to assess our sample and, along with our pathology and cytology lab, perform the correct procedures in order to accurately use our sample. A cytology and pathology lab should work closely together in order to evaluate a biopsy material from a needle sample. Cell blocks produced from a needle biopsy are usually the best solution to evaluate both the cells and the tissue that are acquired with small G needles, like the 21 and $22 \mathrm{G}$. We should use as little material as possible from one paraffin block to identify the cancer type so that we still have enough material left over to evaluate the different genes that might be expressed.

It is very important to make the biopsy as convenient and safe as possible for a patient. For endoscopic techniques, sedation should always be used. There are cases where biopsies in small lymph nodes $(\geq 5 \mathrm{~mm})$ are required for proper lung cancer staging, and general anesthesia is necessary. Regarding techniques such as cone beam, transthoracic ultrasound and CT-guided biopsy, local anesthesia with lidocaine or xylocaine is enough. The status of the respiratory system is very important to consider when evaluating what type of anesthesia should be administered along with the proper biopsy method. Jet-ventilation is a respiratory model used where the respiratory rate is very high $(>60)$ along with a very high tidal volume $(>450)$. A catheter first enters the vocal cords and then the trachea while the patient is in deep sedation but is not intubated [16]. This model of respiration during an interventional procedure is excellent for patients with chronic obstruction pulmonary disease, because these patients tend to experience rapid increases in $\mathrm{PCO}_{2}$ during an endoscopic procedure. Positron emission computed tomography can indicate where to puncture within a large mass in order to obtain live cancer cells. Large masses, especially squamous cell carcinoma, tend to experience tumor necrosis and develop abscesses, meaning false-negative results are often given. This imaging technique provides information regarding the staging of lung cancer, it identifies suspicious lymph nodes of the mesothorax and provides us with the first information of the extent of the disease until the CP-EBUS verifies this information. Medical thoracoscopy is another technique which a pulmonary physician or a thoracic surgeon can perform. We can easily visualize the pleura and perform large tissue biopsies and, if necessary, perform pleurodesis [17]. Rapid on-site evaluation is another technique that provides information within the biopsy procedure, regarding whether the material that we obtained was sufficient for diagnosis. Moreover, it 
provides information on whether we have lung cancer in the site that we punctured; small cell or non-small-cell lung cancer, or distant metastasis. This technique has its limitations, since we might have false negative in cases of lymphoma [15]. Whenever we suspect lymphoma, thoracoscopy should be preferred. Large 19G core biopsies from endoscopic techniques might sometimes provide us with the diagnosis of lymphoma but we still have false-negative results in some patients $[18,19]$.

Another noninvasive method of rapid on-site evaluation is elastography, in which we use the color and strain ration in order to have a real-time tissue evaluation [20]. It allows us to know if a lesion or a lymph node is benign or malignant and where exactly to puncture within the lesion in order to obtain the best sample. When drug resistance occurs with disease relapse in patients that are EGFR positive and are under TKIs, we need to obtain a new sample, either tissue or cytology, to investigate whether we have T790M mutation. Current medical opinion suggests that we should re-biopsy the cancer site if it relapses, if a new lesion occurs or if a lymph node is enlarged [2,21]. Finally, the method of biopsy should be chosen based on the safety of the patient and our first choice should be to obtain tissue sample.

Financial \& competing interests disclosure

The authors have no relevant affiliations or financial involvement with any organization or entity with a financial interest in or financial conflict with the subject matter or materials discussed in the manuscript. This includes employment, consultancies, honoraria, stock ownership or options, expert testimony, grants or patents received or pending, or royalties.

No writing assistance was utilized in the production of this manuscript.

\section{Open access}

This work is licensed under the Attribution-NonCommercial-NoDerivatives 4.0 Unported License. To view a copy of this license, visit http://creativecommons.org/licenses/by-nc-nd/4.0/

\section{References}

1. Domvri K, Zarogoulidis P, Darwiche K et al. Molecular targeted drugs and biomarkers in NSCLC, the evolving role of individualized therapy. J. Cancer 4(9), 736-754 (2013).

2. Zarogoulidis P, Rapti A, Sardeli C et al. Re-biopsy after relapse of targeted therapy. T790M after epidermal growth factor mutation, where and why based on a case series. Respir. Med. Case Rep. 21, 171-175 (2017).

3. Sundaresan TK, Sequist LV, Heymach JV et al. Detection of T790M, the acquired resistance EGFR mutation, by tumor biopsy versus noninvasive blood-based analyses. Clin. Cancer Res. 22(5), 1103-1110 (2016).

4. Domvri K, Darwiche K, Zarogoulidis P, Zarogoulidis K. Following the crumbs: from tissue samples, to pharmacogenomics, to NSCLC therapy. TLCR 2(4), 256-258 (2013).

5. Tsoulos N, Papadopoulou E, Metaxa-Mariatou V et al. Tumor molecular profiling of NSCLC patients using next generation sequencing. Oncol. Rep. 38(6), 3419-3429 (2017).

6. Zarogoulidis K, Zarogoulidis P, Darwiche K et al. Treatment of non-small-cell lung cancer (NSCLC). J. Thorac. Dis. 5(Suppl. 4), S389-S396 (2013).

7. Dafni U, Tsourti Z, Vervita K, Peters S. Immune checkpoint inhibitors, alone or in combination with chemotherapy, as first-line treatment for advanced non-small-cell lung cancer. A systematic review and network meta-analysis. Lung Cancer 134, 127-140 (2019).

8. Zarogoulidis $\mathrm{P}$, Huang H, Tsiouda T et al. Immunotherapy "shock" with vitiligo due to nivolumab administration as third line therapy in lung adenocarcinoma. Respir. Med. Case Rep. 22, 283-286 (2017).

9. Zarogoulidis P, Chinelis P, Athanasiadou A et al. Possible adverse effects of immunotherapy in non-small-cell lung cancer; treatment and follow-up of three cases. Respir. Med. Case Rep. 22, 101-105 (2017).

10. Zarogoulidis P, Athanasiou E, Tsiouda T et al. Immunotherapy "shock" a case series of PD-L1 100\% and pembrolizumab first-line treatment. Respir. Med. Case Rep. 22, 197-202 (2017).

11. Zaric B, Stojsic V, Sarcev T et al. Advanced bronchoscopic techniques in diagnosis and staging of lung cancer. J. Thorac. Dis. 5(Suppl. 4), S359-S370 (2013).

12. Zaric B, Stojsic V, Carapic V et al. Radial endobronchial ultrasound (EBUS) guided suction catheter-biopsy in histological diagnosis of peripheral pulmonary lesions. J. Cancer 7(1), 7-13 (2016).

13. Hohenforst-Schmidt W, Zarogoulidis P, Vogl T et al. Cone beam computertomography (CBCT) in interventional chest medicinehigh feasibility for endobronchial realtime navigation. J. Cancer. 5(3), 231-241 (2014).

14. Hohenforst-Schmidt W, Banckwitz R, Zarogoulidis P et al. radiation exposure of patients by cone beam CT during endobronchial navigation - a phantom study. J. Cancer 5(3), 192-202 (2014). 
15. Oezkan F, Khan A, Zarogoulidis P et al. Efficient utilization of EBUS-TBNA samples for both diagnosis and molecular analyses. Onco. Targets Ther. 7, 2061-2065 (2014).

16. Hohenforst-Schmidt W, Zarogoulidis $\mathrm{P}, \mathrm{Huang} \mathrm{H}$ et al. A new and safe mode of ventilation for interventional pulmonary medicine: the ease of nasal superimposed high frequency jet ventilation. J. Cancer 9(5), 816-833 (2018).

17. Yap KH, Phillips MJ, Lee YC. Medical thoracoscopy: rigid thoracoscopy or flexi-rigid pleuroscopy? Curr. Opin. Pulm. Med. 20(4), 358-365 (2014).

18. Yu L, Chen $\mathrm{K}, \mathrm{Xu} \mathrm{Y}$ et al. The value of EUS in combination with cytological, flow cytometry, and gene rearrangement in the diagnosis of gastrointestinal lymphoma. Hematol. Oncol. 35(3), 303-309 (2017).

19. Zarogoulidis P, Huang H, Bai C et al. Endobronchial ultrasound convex probe for lymphoma, sarcoidosis, lung cancer and other thoracic entities. A case series. Respir. Med. Case Rep. 22, 187-196 (2017).

20. Huang $\mathrm{H}$, Huang $\mathrm{Z}$, Wang $\mathrm{Q}$ et al. Effectiveness of the benign and malignant diagnosis of mediastinal and hilar lymph nodes by endobronchial ultrasound elastography. J. Cancer 8(10), 1843-1848 (2017).

21. Zarogoulidis P, Gaga M, Huang H, Darwiche K, Rapti A, Hohenforst-Schmidt W. Tissue is the issue and tissue competition. Re-biopsy for mutation T790: where and why? Clin. Transl. Med. 6(1), 6 (2017). 